\title{
Initialization of ice-sheet forecasts viewed as an inverse Robin problem
}

\author{
Robert J. ARTHERN, G. Hilmar GUDMUNDSSON \\ British Antarctic Survey, Natural Environment Research Council, Madingley Road, Cambridge CB3 OET, UK \\ E-mail: rart@bas.ac.uk
}

\begin{abstract}
As simulations of 21st-century climate start to include components with longer timescales, such as ice sheets, the initial conditions for those components will become critical to the forecast. This paper describes an algorithm for specifying the initial state of an ice-sheet model, given spatially continuous observations of the surface elevation, the velocity at the surface and the thickness of the ice. The algorithm can be viewed as an inverse procedure to solve for the viscosity or the basal drag coefficient. It applies to incompressible Stokes flow over an impenetrable boundary, and is based upon techniques used in electric impedance tomography; in particular, the minimization of a type of cost function proposed by Kohn and Vogelius. The algorithm can be implemented numerically using only the forward solution of the Stokes equations, with no need to develop a separate adjoint model. The only requirement placed upon the numerical Stokes solver is that boundary conditions of Dirichlet, Neumann and Robin types can be implemented. As an illustrative example, the algorithm is applied to shear flow down an impenetrable inclined plane. A fully three-dimensional test case using a commercially available solver for the Stokes equations is also presented.
\end{abstract}

\section{INTRODUCTION}

We consider a new approach to initializing simulations of ice sheets. Our focus is on simulations used for prediction of 21 st-century climate and sea level.

A close analogy can be drawn between ice-sheet prediction and weather forecasting. For a short-term weather forecast, the initial state of the atmosphere is a critical piece of information and must be derived from observations; the model equations are then integrated numerically to predict how the state will evolve. By contrast, in longer-term simulations of 21 st-century climate, the precise specification of the initial state has so far been considered relatively unimportant. This is because only the statistics of future climate are sought, not details of the actual storms and weather events that will occur decades from now. Taking this statistical point of view, errors in the initial state of the atmosphere decorrelate and become unimportant after a few days or weeks.

Because ice sheets change more slowly than the atmosphere, predicting their behaviour over the coming century has more in common with short-term weather prediction: small errors in the initial state of an ice sheet could systematically affect a climate forecast throughout the $21 \mathrm{st}$ century, perhaps even growing unstably to dominate the predicted sea-level contribution. The ocean circulation in any coupled simulation that includes ice sheets would also be affected. Without a realistic initial configuration, models will not simulate the evolution of the ice sheets accurately, and will give poor predictions of freshwater forcing of ocean circulation and sea-level rise over the coming century.

Initializing an ice-sheet model by a period of 'spinup' over many thousands of years takes little account of the available observations, and is therefore unlikely to represent the current geometry and flow adequately. The present-day geometry of the ice sheets is known fairly accurately from satellite and airborne remote-sensing methods. The horizontal component of the surface velocity can be estimated from satellite radar interferometry and other methods (e.g. Goldstein and others, 1993). The vertical component of velocity can be inferred from the downslope motion combined with satellite radar altimetry (e.g. Wingham and others, 2006) and observations of the rate of snow accumulation on the ice sheet (e.g. Arthern and others, 2006). Here, we propose a simple algorithm that can make use of these data to initialize an ice-sheet model.

An initialization scheme, based around a linearized shallow-ice model, has been described previously (Arthern and Hindmarsh, 2006). One motivation for this study is to take advantage of better measurements and interpolation methods by improving the description of ice flow. Basal drag caused by sliding is considered, and all components of the stress tensor are retained, including membrane stresses caused by lateral extension, compression and shearing (e.g. Hindmarsh, 2006).

Throughout this paper we assume that continuous fields of surface elevation, ice thickness and surface velocity are available at the time of model initialization. Methods for the spatio-temporal interpolation of scattered observations of this type have been developed (e.g. Arthern and Hindmarsh, 2003). In this context, continuous approximations to the required fields can be estimated, albeit subject to some level of measurement and interpolation error.

Despite the available observations, several sources of uncertainty remain in specifying the initial state of the ice sheet. Chief among these is the drag coefficient that determines the slipperiness of the lubricating sediment beneath the ice. This relates the basal stress to the basal velocity. Another source of uncertainty is the relationship between velocity and stresses near the grounding line, where ice becomes afloat as it reaches the ocean: forces imposed by laterally confined, floating ice shelves, or poorly mapped sediment wedges, are not accurately known. A third significant source of uncertainty, despite useful information from laboratory experiments, is the viscosity of the ice sheet. This depends upon the temperature of the ice, and its crystal fabric, and these are not well defined from observations. The 


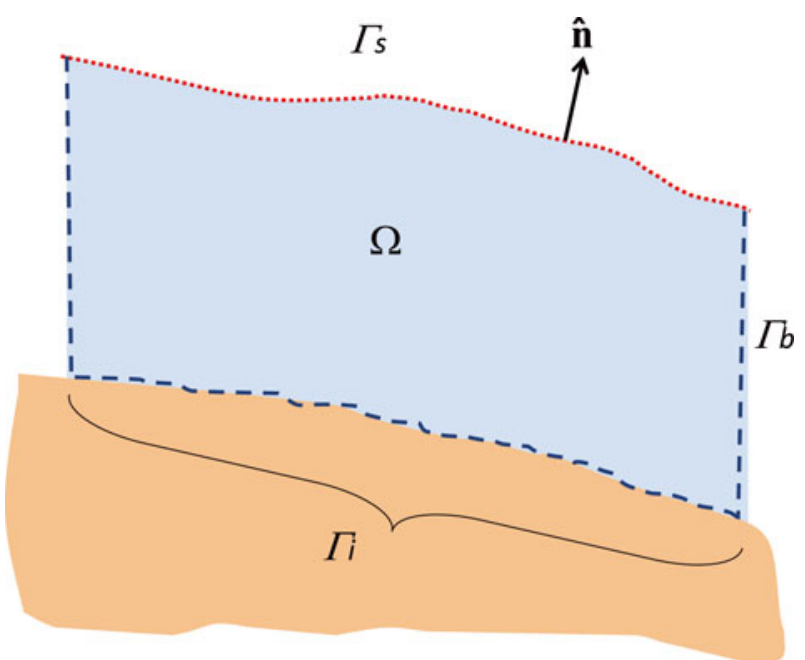

Fig. 1. Geometric depiction of the problem. The ice volume, $\Omega$, has known shape. Measurements of the surface velocity and the stress are available on $\Gamma_{\mathrm{s}}$ (red dotted). On the rest of the boundary, $\Gamma_{\mathrm{b}}$ (blue dashed), including the impenetrable part, $\Gamma_{\mathrm{i}}$, stresses are defined by a Robin-type boundary condition, with unknown Robin coefficient, $\beta$. The viscosity, $\mu$, within $\Omega$ is also unknown. We seek solutions for $\beta$ on $\Gamma_{\mathrm{b}}$, and $\mu$ within $\Omega$, so that velocities and stresses on $\Gamma_{\mathrm{s}}$ are consistent with the observational data.

temperature can also affect basal drag enormously, especially when the bed makes the transition from melted to frozen, or vice versa.

In this paper, we treat the drag coefficient as an undetermined coefficient in a Robin (or 'third kind') boundary condition (Gustafson and Abe, 1998; Gustafson, 1999). The Robin condition may be viewed as a linear combination of Dirichlet (velocity) and Neumann (stress) boundary conditions; each is related to the other via the drag coefficient. Here we present a new algorithm to solve for the basal drag coefficient, $\beta$, and the viscosity, $\mu$. More generally, the approach solves for any spatially variable Robin coefficient, and could perhaps be used to parameterize stresses near the grounding line.

The algorithm can be viewed as a generalization of the inverse method proposed by MacAyeal $(1992,1993)$, which has been applied successfully to many ice streams and ice shelves. However, the new algorithm should address a wider class of problems than either the shallow-ice or other asymptotically approximated models (e.g. Muszynski and Birchfield, 1987; MacAyeal, 1989), including the initialization of higher-order models of ice flow (e.g. Pattyn and others, 2008), and the full system of Stokes equations.

A practical advantage of the new approach is that it can be implemented numerically using only the forward solver of the Stokes equations, with no need to develop a separate adjoint model. The only requirement placed upon the solver is that boundary conditions of Dirichlet, Neumann and Robin types (i.e. fixed velocity, fixed stress and linearly related stress and velocity) can be implemented in the momentumconservation equation. In this respect it is similar to a recent algorithm proposed by Maxwell and others (2008), which has been applied to a transverse cross section of a glacier.

Our algorithm differs from that of Maxwell and others (2008) in several respects. The problem is viewed as an 'inverse Robin problem' (Chaabane and Jaoua, 1999). This is translated into a variational problem through the use of a particular form of cost function, first introduced by Kohn and Vogelius (1984). We provide an explicit expression for the gradient of this cost function, and show that this gradient can be calculated efficiently. An analogous problem, in the context of electric impedance tomography, has been studied by Chaabane and Jaoua (1999) for the Laplace equation. The analysis and notation used in this paper closely follows theirs.

Here, we formulate an inverse Robin problem for Stokes flow as commonly encountered in glaciological applications. We also describe an algorithm for the numerical solution of the problem. As a proof of concept, we describe synthetic inversion of basal properties: first for an illustrative onedimensional (1-D) example; second for a more realistic, three-dimensional (3-D) example, with nonlinear ice and till rheology. In the latter example we use a commercially available solver for the Stokes equations.

\section{THEORY}

Assume the ice sheet occupies a volume, $\Omega$, of known shape, as shown in Figure 1, bounded by a surface, $\Gamma_{\mathrm{s}}$, where we have observations, and a surface, $\Gamma_{b}$, where we wish to solve for the coefficient that linearly relates stresses on the boundary to velocities there, i.e. the parameter in a Robintype boundary condition. Using Cartesian coordinates, $(x, y, z)$, denote the outward normal vector on both surfaces by $\hat{\mathbf{n}}$, the material velocity by $\mathbf{v}$, strain-rate tensor by $\dot{\mathbf{e}} \equiv$ $\frac{1}{2}\left[(\nabla \mathbf{v})+(\nabla \mathbf{v})^{*}\right]$, stress tensor by $\boldsymbol{\sigma}$, and pressure by $p \equiv$ - Trace $(\boldsymbol{\sigma}) / 3$. For now, assume an incompressible material, so that $\nabla \cdot \mathbf{v}=0$, and a linear viscous rheology, so deviatoric stress is given by $\boldsymbol{\sigma}+p \mathbf{I}=2 \mu \dot{\mathbf{e}}$, with identity $\mathbf{I}$, and viscosity $\mu$. Further assuming a constant density, $\rho$, and gravitational field, $\mathbf{g}$, the momentum-conservation equation corresponding to force balance can be written $\nabla \cdot \boldsymbol{\sigma}+\rho \mathbf{g}=0$.

To begin with, consider a simplified problem, in which the force per unit area on the boundary, $\Gamma_{b}$, acts in the opposite direction to the velocity, and in proportion to it, so that $\boldsymbol{\sigma} \cdot \hat{\mathbf{n}}+\beta \mathbf{v}=0$, with $\beta>0$, a scalar that may vary spatially; this relationship defines the Robin boundary condition. Later we can adapt this to the more realistic case of sliding past an impenetrable boundary, $\Gamma_{\mathrm{i}}$, that supports normal and tangential stresses, such as the base of the ice sheet, but for now we ignore the distinction between $\Gamma_{i}$ and the rest of $\Gamma_{\mathrm{b}}$.

For the simplified problem the initialization consists of selecting viscosity, $\mu$, in the interior of the domain, and the Robin parameter, $\beta$, on the boundary. The inverse Robin problem $(\mathcal{I P})$ can be stated as follows:

$$
\begin{aligned}
& \text { (Given the prescribed boundary stress, } \boldsymbol{\tau} \text {, } \\
& \text { and velocity measurements, } \mathbf{f} \text {, on } \Gamma_{\mathrm{s}} \text {, } \\
& \text { find functions } \beta \text { on } \Gamma_{\mathrm{b}} \text {, and } \mu \text { in } \Omega \text {, } \\
& \text { such that the solution, } \mathbf{v} \text {, of } \\
& (\mathcal{I P})
\end{aligned}
$$

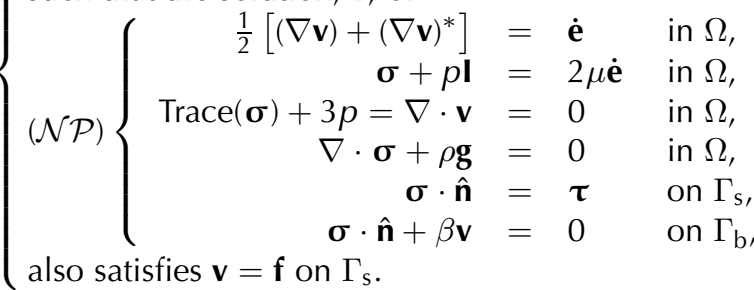

We assume the availability of an ice-sheet model, optimized for the efficient solution of the Neumann problem 
$(\mathcal{N} \mathcal{P})$, and that this model can also be used to solve a Dirichlet problem $(\mathcal{D} \mathcal{P})$, forced by the surface velocity measurements, $\mathbf{f}$ :

$$
(\mathcal{D P})\left\{\begin{aligned}
\frac{1}{2}\left[(\nabla \mathbf{v})+(\nabla \mathbf{v})^{*}\right] & =\mathbf{e} & & \text { in } \Omega, \\
\boldsymbol{\sigma}+p \mathbf{I} & =2 \mu \dot{\mathbf{e}} & & \text { in } \Omega, \\
\operatorname{Trace}(\boldsymbol{\sigma})+3 p=\nabla \cdot \mathbf{v} & =0 & & \text { in } \Omega, \\
\nabla \cdot \boldsymbol{\sigma}+\rho \mathbf{g} & =0 & & \text { in } \Omega, \\
\mathbf{v} & =\mathbf{f} & & \text { on } \Gamma_{\mathrm{s}} \\
\boldsymbol{\sigma} \cdot \hat{\mathbf{n}}+\beta \mathbf{v} & =0 & & \text { on } \Gamma_{\mathrm{b}} .
\end{aligned}\right.
$$

Writing the solutions of the Dirichlet and Neumann problems $\mathcal{D P}$ and $\mathcal{N} \mathcal{P}$ as $\left[\mathbf{v}^{\mathrm{D}}, \dot{\mathbf{e}}^{\mathrm{D}}, \boldsymbol{\sigma}^{\mathrm{D}}, p^{\mathrm{D}}\right]$ and $\left[\mathbf{v}^{\mathrm{N}}, \dot{\mathbf{e}}^{\mathrm{N}}, \boldsymbol{\sigma}^{\mathrm{N}}, p^{\mathrm{N}}\right]$, respectively, the Appendix shows that the inverse problem $(\mathcal{I P})$ can be solved by minimizing the Kohn and Vogelius (1984) cost function

$$
J(\mu, \beta)=\int_{\Omega} 2 \mu\left|\dot{\mathbf{e}}^{\mathrm{N}}-\dot{\mathbf{e}}^{\mathrm{D}}\right|_{\mathrm{F}}^{2}+\int_{\Gamma_{\mathrm{b}}} \beta\left|\mathbf{v}^{\mathrm{N}}-\mathbf{v}^{\mathrm{D}}\right|_{\mathrm{F}}^{2},
$$

in which the subscript ' $F$ ' denotes the Frobenius norm, as defined in the Appendix. Furthermore, the gradient of this cost function, with respect to the parameters $\mu$ and $\beta$, can be expressed as a Gâteaux, or directional, derivative (e.g. Zorn, 1946), defined by $\mathrm{d} J \equiv \mathrm{d}_{\mu} J+\mathrm{d}_{\beta} J$, with

$$
\begin{aligned}
\mathrm{d}_{\mu} J\left(\mu, \beta, \mu^{\prime}\right) & \equiv \lim _{\varepsilon \rightarrow 0^{+}} \frac{J\left(\mu+\varepsilon \mu^{\prime}, \beta\right)-J(\mu, \beta)}{\varepsilon} \\
& =\int_{\Omega} 2 \mu^{\prime}\left(\left|\dot{\mathbf{e}}^{\mathrm{D}}\right|_{\mathrm{F}}^{2}-\left|\dot{\mathbf{e}}^{\mathrm{N}}\right|_{\mathrm{F}}^{2}\right) ; \\
\mathrm{d}_{\beta} J\left(\mu, \beta, \beta^{\prime}\right) & \equiv \lim _{\varepsilon \rightarrow 0^{+}} \frac{J\left(\mu, \beta+\varepsilon \beta^{\prime}\right)-J(\mu, \beta)}{\varepsilon} \\
& =\int_{\Gamma_{\mathrm{b}}} \beta^{\prime}\left(\left|\mathbf{v}^{\mathrm{D}}\right|_{\mathrm{F}}^{2}-\left|\mathbf{v}^{\mathrm{N}}\right|_{\mathrm{F}}^{2}\right),
\end{aligned}
$$

where $\varepsilon$ is a small parameter that tends to zero in the limit. A more realistic case is that some part of the boundary, $\Gamma_{b}$, is impenetrable, such as the base of the ice sheet. On the impenetrable part, $\Gamma_{\mathrm{i}}$, conditions of zero normal velocity, and tangential shear stress proportional to velocity, can be applied:

$$
\begin{aligned}
\mathbf{v} \cdot \hat{\mathbf{n}} & =0 & & \text { on } \Gamma_{\mathrm{i}} \\
(\mathbf{I}-\hat{\mathbf{n}} \hat{\mathbf{n}}) \cdot \boldsymbol{\sigma} \cdot \hat{\mathbf{n}}+\beta \mathbf{v} & =0 & & \text { on } \Gamma_{\mathrm{i}} .
\end{aligned}
$$

Repeating the analysis with this boundary condition, the product of normal forces and normal velocities vanishes in surface integrals over $\Gamma_{\mathrm{i}}$, and the results are identical to Equations (3) and (4). Thus, the inverse Robin problem for the case of sliding past an impenetrable boundary can be solved by minimizing $J$, defined by Equation (3), subject to constraints $\mathcal{D P}$ and $\mathcal{N} \mathcal{P}$, as modified by Equation (5). Furthermore, Equation (4) continues to provide the Gâteaux derivative of $J$ in that case.

\section{METHODS}

By analogy with the investigation of the Laplace equation, described by Chaabane and Jaoua (1999), the above theory suggests an iterative descent algorithm for minimizing $J$, and hence selecting $\mu$ and $\beta$ :
Algorithm 1
1. Given initial estimates $\mu_{0}$ and $\beta_{0}$.
2. Solve $\mathcal{N P}$ to obtain $\mathbf{v}^{\mathrm{N}}$ and $\dot{\mathbf{e}}^{\mathrm{N}}$.
3. Solve $\mathcal{D P}$ to obtain $\mathbf{v}^{\mathrm{D}}$ and $\dot{\mathbf{e}}^{\mathrm{D}}$.
4. For stepsize parameters $\alpha_{\mu}>0$ and $\alpha_{\beta}>0$.
5. Update $\mu_{n+1} \rightarrow \mu_{n}-\alpha_{\mu}\left(\left|\dot{\mathbf{e}}^{\mathrm{D}}\right|_{\mathrm{F}}^{2}-\left|\dot{\mathbf{e}}^{\mathrm{N}}\right|_{\mathrm{F}}^{2}\right)$ in $\Omega$.
6. Update $\beta_{n+1} \rightarrow \beta_{n}-\alpha_{\beta}\left(\left|\mathbf{v}^{\mathrm{D}}\right|_{\mathrm{F}}^{2}-\left|\mathbf{v}^{\mathrm{N}}\right|_{\mathrm{F}}^{2}\right)$ on $\Gamma_{\mathrm{b}}$.
7. Return to Step 2 unless converged.

To clarify the above notation, at Step 5 the estimated viscosity, $\mu$, is reduced by an amount $\alpha_{\mu}\left(\left|\dot{\mathbf{e}}^{\mathrm{D}}\right|_{\mathrm{F}}^{2}-\left|\dot{\mathbf{e}}^{\mathrm{N}}\right|_{\mathrm{F}}^{2}\right)$, where the strain-rate tensors, $\dot{\mathbf{e}}^{\mathrm{D}}$ and $\dot{\mathbf{e}}^{\mathrm{N}}$, are available from the Dirichlet and Neumann simulations, respectively.

\section{RESULTS}

For the purpose of illustration, consider the following simple application of Algorithm 1, estimating the basal drag coefficient, $\beta$, and viscosity, $\mu$, for an infinite slab of constant thickness, $h$, sliding down an impenetrable, inclined plane at angle $\alpha$ from the horizontal. Define tilted rectangular coordinates so the $x$ - and $y$-axes are within the plane (in the down- and across-slope directions, respectively) and the $z$ axis is normal to the plane, increasing upwards. Then neglect all variation in $x$ and $y$. For observational constraints, specify the downslope surface velocity, $f$, in the $x$ direction and that the shear stress, $\sigma_{x z}=\mu \partial_{z} v$, vanishes at the surface. For this problem the Neumann and Dirichlet problems are second-order, 1-D, boundary-value problems, and can be written in nondimensional form by defining scales $|z|=h$, $|v|=f,|\boldsymbol{\tau}|=\rho g h \sin \alpha,|\mu|=|\boldsymbol{\tau}| h / f,|\beta|=|\boldsymbol{\tau}| / f$. In these units, conservation of momentum in the $x$ direction gives

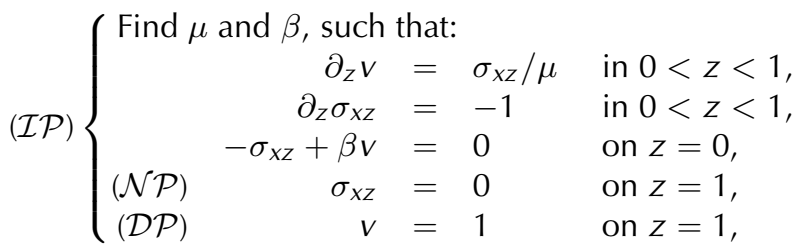

where unlabelled equations correspond to both Dirichlet $(\mathcal{D} \mathcal{P})$ and Neumann $(\mathcal{N} \mathcal{P})$ problems. Further assuming no variation of $\mu$ within the slab, so the parameter space defined by $\mu$ and $\beta$ is two-dimensional, the cost function, J, can be plotted. Figure 2 shows the cost function. This was obtained by numerically solving the boundary-value problems $\mathcal{N} \mathcal{P}$ and $\mathcal{D P}$ (Equation (7)) for regularly spaced $\mu$ and $\beta$ (using MATLAB routine bvp4c; Kierzenka and Shampine, 2001), then evaluating J using Equation (3). The figure also shows the result of applying Algorithm 1, from various initial estimates, with the boundary-value problems solved numerically at each step of the algorithm.

In a more realistic application, with multiple dimensions, it would be impractical to plot the cost function, and specialized numerical algorithms for solving the full Stokes system, or some approximation to it, would be employed in the descent algorithm. Nevertheless, this simple example illustrates the main features of the method. 


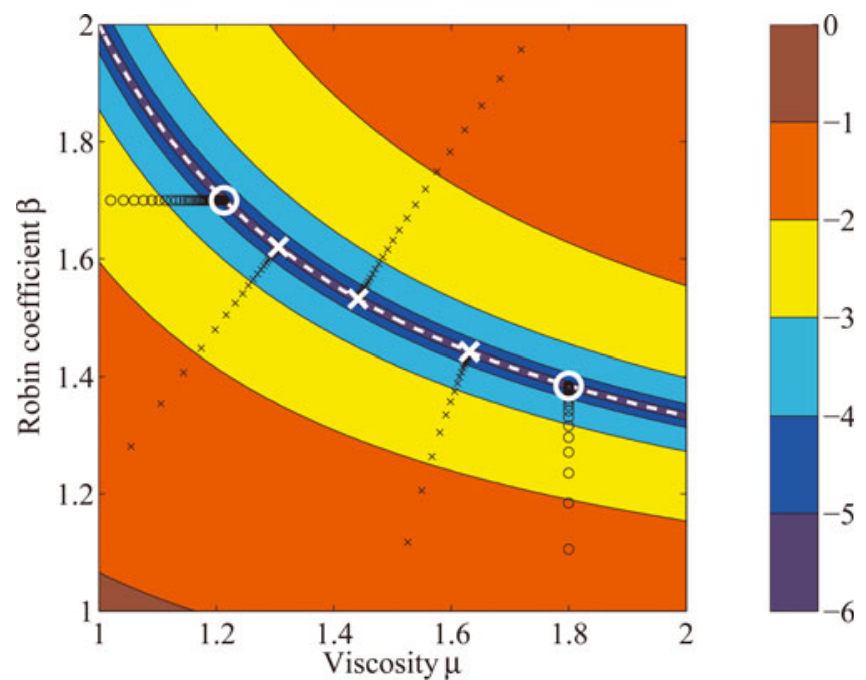

Fig. 2. Results of applying Algorithm 1 to retrieve the viscosity, $\mu$, and basal drag coefficient, $\beta$, for shear flow down an inclined plane. Crosses show the iterative path taken to minimize the cost function, $J(\mu, \beta)$, from various initial estimates. The coloured contours show $\log _{10} \mathrm{~J}$. The white dashed curve shows the analytical solution $\beta=2 \mu /(2 \mu-1)$. In general, no unique solution is obtained unless an independent estimate of $\mu$ or $\beta$ is available. Circles show two such examples, constrained by prior estimates $\mu=1.8$ and $\beta=1.7$.

In fact, the above example can be integrated directly, to reveal that there is no unique solution for nondimensional $\mu$ and $\beta$ which solves the inverse problem $\mathcal{I} \mathcal{P}$, but rather a locus of solutions defined by $\beta=2 \mu /(2 \mu-1)$. This locus is shown as a white dashed curve in Figure 2. As anticipated from the theory, it corresponds closely to the minimum of the cost function, and to the solutions converged upon by Algorithm 1.

Figure 2 illustrates that to solve for the drag coefficient, $\beta$, prior information about the viscosity, $\mu$, is required. Similarly, to solve for $\mu$ requires prior information about $\beta$. Nevertheless, if $\mu$ (or $\beta$ ) is already known, perhaps from laboratory experiments, Algorithm 1 can be used to determine the other, by setting the initial estimate $\mu_{0}$ (or $\beta_{0}$ ) to the known value, and the corresponding descent parameter $\alpha_{\mu}$ (or $\alpha_{\beta}$ ) to zero: examples are shown in Figure 2 .

A more realistic test of the algorithm is shown in Figure 3. This shows a synthetic inversion in a 3-D setting, with nonlinear viscosities for both ice and basal sediment (till). Synthetic data were generated using a commercial Stokes solver to simulate flow down an ice stream $100 \mathrm{~km}$ long, $30 \mathrm{~km}$ wide and $1.6 \mathrm{~km}$ thick, over an isolated patch of lowviscosity till (Fig. 3a). The slope was 0.01 with elevation decreasing in the $x$ direction, driving flow from left to right in Figure 3.

Ice rheology was prescribed by a nonlinear Glen flow law $\left(\boldsymbol{\sigma}+p \mathbf{I}=A^{-\frac{1}{n}}\left|\frac{\mathbf{1}}{\mathbf{2}} \dot{\mathbf{e}}\right|_{\mathrm{F}}^{\left(\frac{1}{n}-1\right)} \dot{\mathbf{e}}\right)$ with $n=3$ and rate constant $A=4.6 \times 10^{-10} \mathrm{~d}^{-1} \mathrm{kPa}^{-3}$. The upper surface was stress-free in all Neumann computations. Periodic boundary conditions were applied at the horizontal boundaries. At the base, a Weertman-type nonlinear sliding law, $v_{\mathrm{b}}=C \tau_{\mathrm{b}}^{m}$, with $m=3$ was used, with $\tau_{\mathrm{b}}$ representing basal shear stress and $v_{\mathrm{b}}$ the basal flow speed.

The coefficient $C$ was specified by adding a Gaussian perturbation to a constant background level (Fig. 3a).

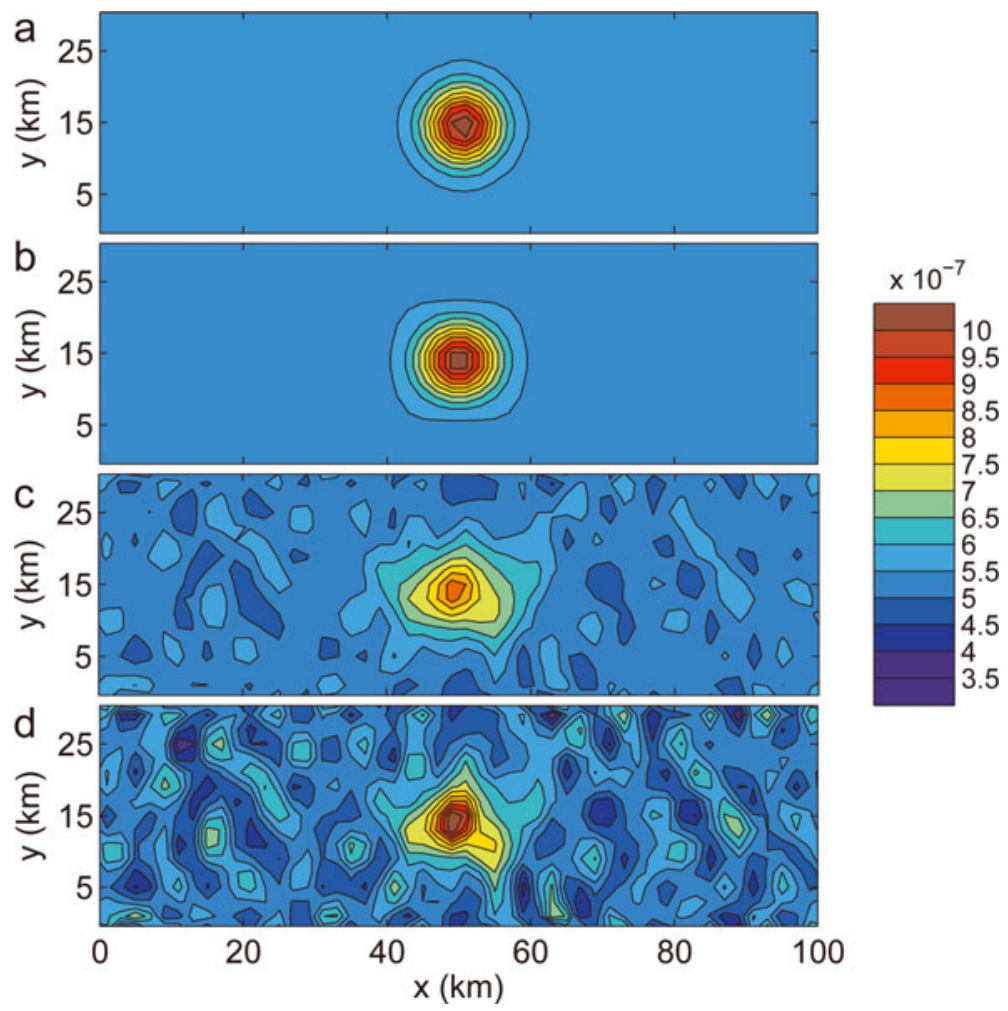

Fig. 3. Results of applying Algorithm 1 to retrieve the rate coefficient for a nonlinear sliding law in a 3-D simulation. A commercial solver for the Stokes equations was used in this inversion. Each panel represents an ice stream $(100 \mathrm{~km} \times 30 \mathrm{~km})$ flowing from left to right over a patch of lower-viscosity sediment. (a) The specified rate coefficient $\left(\mathrm{m} \mathrm{d}^{-1} \mathrm{kPa}^{-3}\right.$ ); (b) the rate coefficient recovered from noise-free synthetic observations that would be available at the surface; (c) the rate coefficient recovered from synthetic surface observations with random noise added to represent measurement errors after 9 iteration steps and (d) after 19 iterations. 
This perturbation, centred on $x=50 \mathrm{~km}, y=15 \mathrm{~km}$, had amplitude equal to the background value $(5.3 \times$ $10^{-7} \mathrm{~m} \mathrm{~d}^{-1} \mathrm{kPa}^{-3}$ ), so that $C$ is double the background value at its peak, with an e-fold decay radius of $5 \mathrm{~km}$.

The rate coefficient, $C$, was then recovered by applying Algorithm 1, using only those data available at the surface. In this experiment, $A$ is assumed known, and no attempt is made to recover a spatially varying viscosity in the interior. The initial guess for $C$ was spatially uniform at the unperturbed background value. The rate coefficient at each location was updated iteratively, from $C_{n}$ to $C_{n+1}$, by adding a contribution in the direction that reduces the cost function. Because an increase in $C$ in effect corresponds to a reduction in $\beta$, a change of sign was made to Step 6 of Algorithm 1, replacing it as follows: $C_{n+1} \rightarrow C_{n}+\alpha_{C}\left(\left|\mathbf{v}_{\mathrm{b}}^{\mathrm{D}}\right|_{\mathrm{F}}^{2}-\right.$ $\left.\left|\mathbf{v}_{\mathrm{b}}^{\mathrm{N}}\right|_{\mathrm{F}}^{2}\right)$, with $\alpha_{\mathrm{C}}>0$. The basal velocity vectors, $\mathbf{v}_{\mathrm{b}}^{\mathrm{D}}$ and $\mathbf{v}_{\mathrm{b}}^{\mathrm{N}}$, were from the Dirichlet and Neumann computations, respectively, and their Frobenius norms are simply their magnitude.

If the step-size parameter, $\alpha_{C}$, is too large, there is a risk of overshooting the minimum, even if the search direction points locally downhill. We set $\alpha_{C}=10^{-7} \mathrm{~m}^{-1}$ day $\mathrm{kPa}^{-3}$, but if a step failed to reduce the cost function, $\alpha_{C}$ was halved and another trial step taken. If this second step failed, the value of $\alpha_{C}$ minimizing $J$ in the current search direction was estimated from a quadratic fit, and $C_{n+1}$ updated using this value.

The extension of our theory to the nonlinear sliding law is heuristic, since we have not differentiated the cost function appropriate to the nonlinear case. Despite this, it works well in practice. Results of the inversion are shown in Figure $3 \mathrm{~b}$. The close match to Figure $3 \mathrm{a}$ demonstrates the successful convergence of the algorithm, even for a 3-D problem with nonlinear rheologies of ice and till.

We investigated the effect of measurement errors upon the algorithm by perturbing the synthetic surface observations with randomly generated noise, before applying the inversion algorithm. Comparing Figure 3a with Figure 3c shows that the recovered rate coefficients suffer some degradation when measurement errors are present. Nevertheless, the inversion is still able to detect the presence of the lowviscosity sediment.

Comparison of Figure $3 c$ with Figure $3 d$ shows that broad-scale features are recovered first. As iterations are continued, the inversion produces sharper features with higher amplitude. Figure 3d shows that this can eventually produce spurious features, by overfitting to measurement errors.

To avoid overfitting, a stopping criterion is needed. For a similar problem Maxwell and others (2008) propose a heuristic criterion based upon the mismatch between Neumann and observed velocities at the surface. Our calculations provide support for this approach. Figure 4 shows the root-mean-square (rms) mismatch between the surface velocity data, $\mathbf{f}$, and the surface velocities computed from the Neumann problem $(\mathcal{N} \mathcal{P})$. For the noise-free test case, the mismatch decreases iteration by iteration. However, when noise is added, the mismatch stagnates at a level consistent with the rms noise level (dashed horizontal line). Thus, a conservative estimate of the observational error on f can serve to define a stopping criterion. As an example, the first iteration to reach the rms noise level is plotted in Figure 3c.

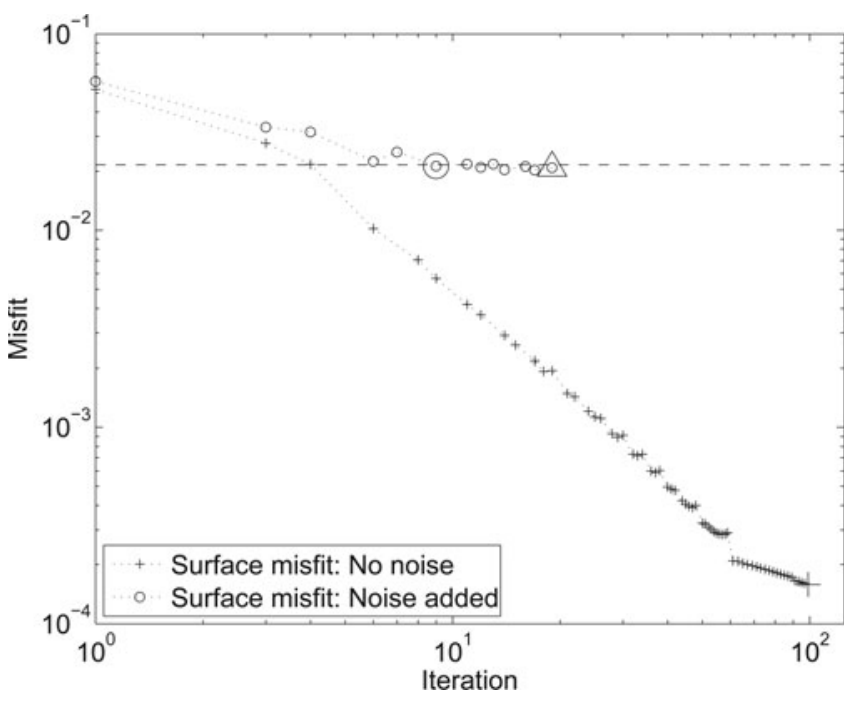

Fig. 4. Convergence for synthetic inversions shown in Figure 3. The plot shows the rms mismatch $\left(\mathrm{m} \mathrm{d}^{-1}\right)$ between Neumann and observed surface velocities. Without measurement errors, convergence continues throughout. When measurement errors are introduced, by adding noise to the synthetic data, the convergence stagnates and a stopping criterion is needed. The horizontal dashed line shows the rms of the added noise. Large symbols show the iterations plotted in Figure 3.

\section{DISCUSSION AND CONCLUSIONS}

For the purpose of initializing ice-sheet forecasts, we have described a simple algorithm to invert for the basal drag coefficient and ice viscosity.

If Algorithm 1 is used to estimate the basal drag coefficient and/or refine the ice viscosity, the ice-sheet forecast will begin in a state of force balance, with present-day geometry and surface velocities. This is preferable to a state beginning with present geometry, but with unrealistic viscosity or drag coefficient, since this would produce an 'initialization shock' at the beginning of the forecast and a spurious contribution to the sea-level forecast.

Use of Algorithm 1 is also preferable to selecting an initial state for a 21 st-century simulation simply by 'spinning up' the ice-sheet model over many thousands of years. An ice-sheet model may have more than a million variables describing its geometry and flow. Without the constraint of observations, there is slim chance of obtaining initial conditions close enough to the present-day values to be useful for prediction.

A practical advantage of Algorithm 1 is its ease of implementation. It only uses the forward solver of the Stokes equations, so there is no requirement to develop and test a separate adjoint model. This also means that Algorithm 1 benefits automatically from any parallelization, adaptive grid refinement or asymptotic approximations employed by the forward model. Conveniently, the terms in Equation (4) are proportional to deformational and frictional heating rates, which are routinely evaluated in many ice-sheet models.

This preliminary investigation does not present an exhaustive test of the ability of Algorithm 1 to recover viscosity and basal drag coefficient from realistic glaciological data. In particular, we have assumed that continuous fields of surface elevation, surface velocity and thickness are available. For some applications, error-prone surface velocity data could be incompatible with incompressibility, perhaps leading the Dirichlet problem to be ill-posed unless this constraint is 
enforced. If the geometry of the bed is poorly known it would be preferable to solve for bed elevation and drag coefficient simultaneously (Gudmundsson and Raymond, 2008; Raymond and Gudmundsson, 2009). This would require modification of our algorithm, and it may be necessary to take account of prior knowledge of the spatial statistics for bed elevation and its uncertainty (Raymond and Gudmundsson, 2009).

To speed convergence, a more sophisticated algorithm, such as conjugate gradients, could be used to minimize the cost function. Deriving the precise derivative of the cost function appropriate for the nonlinear sliding law may also improve the performance. The sensitivity to the stopping criterion suggests that it would be helpful to optimize this aspect further.

Chaabane and others (1999) present a more complete theoretical analysis for the Laplace equation. It would be worth considering which of their results transfer to the glaciological situation considered here. The application to a vector velocity field represents a qualitative difference from the scalar Laplace equation. In particular, further theoretical and numerical work is needed to establish the resolving power of the method, the sensitivity to measurement errors and whether additional regularization is needed. Nevertheless, we hope the inverse Robin problem specified above, the descent algorithm and the preliminary results, will motivate further numerical and theoretical calculations of this kind, so that simulations of 21 st-century climate can be undertaken with properly initialized ice-sheet models.

\section{ACKNOWLEDGEMENTS}

We are grateful to two anonymous reviewers who each suggested a number of improvements. Funding was provided by the Natural Environment Research Council.

\section{REFERENCES}

Arthern, R.J. and R.C.A. Hindmarsh. 2003. Optimal estimation of changes in the mass of ice sheets. J. Geophys. Res., 108(F1), 6007. (10.1029/2003JF000021.)

Arthern, R.J. and R.C.A. Hindmarsh. 2006. Determining the contribution of Antarctica to sea-level rise using data assimilation methods. Philos. Trans. R. Soc. London, Ser. A, 364(1844), 1841-1865.

Arthern, R.J., D.P. Winebrenner and D.G. Vaughan. 2006. Antarctic snow accumulation mapped using polarization of $4.3 \mathrm{~cm}$ wavelength microwave emission. J. Geophys. Res., 111(D6), D06107. (10.1029/2004JD005667.)

Chaabane, S. and M. Jaoua. 1999. Identification of Robin coefficients by the means of boundary measurements. Inverse Probl., 15(6), 1425-1438.

Goldstein, R.M., H. Engelhardt, B. Kamb and R.M. Frolich. 1993. Satellite radar interferometry for monitoring ice sheet motion: application to an Antarctic ice stream. Science, 262(5139), 1525-1530.

Gudmundsson, G.H. and M. Raymond. 2008. On the limit to resolution and information on basal properties obtainable from surface data on ice streams. Cryosphere, 2(1), 167-178.

Gustafson, K.E. 1999. Introduction to partial differential equations and Hilbert space methods. Third edition. New York, Dover Publications.

Gustafson, K. and T. Abe. 1998. The third boundary condition - was it Robin's? Math. Intell., 20(1), 63-71.

Hindmarsh, R.C.A. 2006. The role of membrane-like stresses in determining the stability and sensitivity of the Antarctic Ice
Sheets: back pressure and grounding line motion. Philos. Trans. R. Soc. London, Ser. A, 364(1844), 1733-1767.

Kierzenka, J. and L.F. Shampine. 2001. A BVP solver based on residual control and the MATLAB PSE. ACM Trans. Math. Softw., 27(3), 299-316.

Kohn, R. and M. Vogelius. 1984. Determining conductivity by boundary measurements. Commun. Pure Appl. Math., 37(3), 289-298.

MacAyeal, D.R. 1989. Large-scale ice flow over a viscous basal sediment: theory and application to Ice Stream B, Antarctica. J. Geophys. Res., 94(B4), 4071-4087.

MacAyeal, D.R. 1992. The basal stress distribution of Ice Stream E, Antarctica, inferred by control methods. J. Geophys. Res., 97(B1), 595-603.

MacAyeal, D.R. 1993. A tutorial on the use of control methods in ice-sheet modeling. J. Glaciol., 39(131), 91-98.

Maxwell, D., M. Truffer, S. Avdonin and M. Stuefer. 2008. An iterative scheme for determining glacier velocities and stresses. J. Glaciol., 54(188), 888-898.

Muszynski, I. and G.E. Birchfield. 1987. A coupled marine icestream-ice-shelf model. J. Glaciol., 33(113), 3-15.

Pattyn, F. and 20 others. 2008. Benchmark experiments for higher-order and full-Stokes ice sheet models (ISMIP-HOM). Cryosphere, 2(1), 95-108.

Raymond, M.J. and G.H. Gudmundsson. 2009. Estimating basal properties of ice streams from surface measurements: a nonlinear Bayesian inverse approach applied to synthetic data. Cryosphere, 3(2), 265-278.

Wingham, D.J., A. Shepherd, A. Muir and G.J. Marshall. 2006. Mass balance of the Antarctic ice sheet. Philos. Trans. R. Soc. London, Ser. A, 364(1844), 1627-1635.

Zorn, M.A. 1946. Derivatives and Fréchet differentials. Bull. Am. Math. Soc., 52(2), 133-137.

\section{APPENDIX}

This appendix relates the inverse problem $(\mathcal{I P})$ to the variational problem of minimizing the Kohn and Vogelius (1984) cost function. To identify the minimum of the cost function we write it as an integral over $\Gamma_{\mathrm{S}}$, the surface of the ice sheet, perturb this expression, and apply the fundamental lemma of the calculus of variations. The resulting expression solves the inverse problem $(\mathcal{I P})$. Finally we outline the steps used to derive Equation (4), the gradient of the cost function with respect to small changes in viscosity and basal drag. Velocities, stresses and parameters are implicitly assumed continuous and differentiable throughout.

To simplify notation, define the Frobenius product $\dot{\mathbf{e}}: \boldsymbol{\sigma} \equiv \operatorname{Trace}\left(\dot{\mathbf{e}}^{*} \boldsymbol{\sigma}\right)$, and the Frobenius norm $|\boldsymbol{\sigma}|_{\mathrm{F}}^{2} \equiv$ $\boldsymbol{\sigma}: \boldsymbol{\sigma}$. Generalized superscripts $(A, B) \in\left(D, N, D^{\prime}, N^{\prime}\right)$ denote solutions from either the Neumann $(\mathrm{N})$ or Dirichlet (D) problems, or their first-order perturbations $\left(\mathrm{N}^{\prime}\right.$ and $\mathrm{D}^{\prime}$, respectively, defined later in this appendix). Here are two useful identities: the first, from Leibniz's product rule for differentiation, is

$$
\nabla \cdot \mathbf{v}^{\mathrm{A}} \cdot \boldsymbol{\sigma}^{\mathrm{B}}=\mathbf{v}^{\mathrm{A}} \cdot \nabla \cdot \boldsymbol{\sigma}^{\mathrm{B}}+\dot{\mathbf{e}}^{\mathrm{A}}: \boldsymbol{\sigma}^{\mathrm{B}} ;
$$

the second, from the divergence theorem applied to the volume, $\Omega$, is

$$
\int_{\Omega} \nabla \cdot \mathbf{v}^{\mathrm{A}} \cdot \boldsymbol{\sigma}^{\mathrm{B}}=\int_{\Gamma_{\mathrm{s}}+\Gamma_{\mathrm{b}}} \mathbf{v}^{\mathrm{A}} \cdot \boldsymbol{\sigma}^{\mathrm{B}} \cdot \hat{\mathbf{n}} .
$$

Equations (A1) and (A2), and substitutions from the Neumann and Dirichlet problems $(\mathcal{N P}$ and $\mathcal{D P})$ allow the cost function, $J$, to be written as a surface integral. 


$$
\begin{aligned}
J(\mu, \beta)= & \int_{\Omega} 2 \mu\left|\dot{\mathbf{e}}^{\mathrm{N}}-\dot{\mathbf{e}}^{\mathrm{D}}\right|_{\mathrm{F}}^{2}+\int_{\Gamma_{\mathrm{b}}} \beta\left|\mathbf{v}^{\mathrm{N}}-\mathbf{v}^{\mathrm{D}}\right|_{\mathrm{F}}^{2} \\
= & \int_{\Omega} \nabla \cdot\left(\mathbf{v}^{\mathrm{N}}-\mathbf{v}^{\mathrm{D}}\right) \cdot 2 \mu\left(\dot{\mathbf{e}}^{\mathrm{N}}-\dot{\mathbf{e}}^{\mathrm{D}}\right) \\
& -\int_{\Omega}\left(\mathbf{v}^{\mathrm{N}}-\mathbf{v}^{\mathrm{D}}\right) \cdot \nabla \cdot\left(\boldsymbol{\sigma}^{\mathrm{N}}+p^{\mathrm{N}} \mathbf{I}-\mathbf{\sigma}^{\mathrm{D}}-p^{\mathrm{D}} \mathbf{I}\right) \\
& +\int_{\Gamma_{\mathrm{b}}} \beta\left|\mathbf{v}^{\mathrm{N}}-\mathbf{v}^{\mathrm{D}}\right|_{\mathrm{F}}^{2} \\
= & \int_{\Gamma_{\mathrm{s}}}\left(\mathbf{v}^{\mathrm{N}}-\mathbf{v}^{\mathrm{D}}\right) \cdot\left(\boldsymbol{\sigma}^{\mathrm{N}}-\boldsymbol{\sigma}^{\mathrm{D}}\right) \cdot \hat{\mathbf{n}} \\
& +\int_{\Gamma_{\mathrm{b}}}\left(\mathbf{v}^{\mathrm{N}}-\mathbf{v}^{\mathrm{D}}\right) \cdot\left(\boldsymbol{\sigma}^{\mathrm{N}}-\boldsymbol{\sigma}^{\mathrm{D}}\right) \cdot \hat{\mathbf{n}} \\
& +\int_{\Gamma_{\mathrm{b}}} \beta\left|\mathbf{v}^{\mathrm{N}}-\mathbf{v}^{\mathrm{D}}\right|_{\mathrm{F}}^{2} \\
= & \int_{\Gamma_{\mathrm{s}}}\left(\mathbf{v}^{\mathrm{N}}-\mathbf{v}^{\mathrm{D}}\right) \cdot\left(\boldsymbol{\sigma}^{\mathrm{N}}-\boldsymbol{\sigma}^{\mathrm{D}}\right) \cdot \hat{\mathbf{n}} .
\end{aligned}
$$

Small perturbations, $\mu^{\prime}$ and $\beta^{\prime}$, to $\mu$ and $\beta$ will affect the solutions $\mathbf{v}^{\mathrm{N}}$ and $\mathbf{v}^{\mathrm{D}}$ by small amounts $\mathbf{v}^{\mathrm{N}^{\prime}}$ and $\mathbf{v}^{\mathrm{D}^{\prime}}$. These changes will perturb the cost function, J, by an amount $\mathrm{d} J$. To first order:

$$
\begin{aligned}
\mathrm{d} J=\mathrm{d}_{\mu} J+\mathrm{d}_{\beta} J= & \int_{\Gamma_{\mathrm{s}}}\left(\mathbf{v}^{\mathrm{N}^{\prime}}-\mathbf{v}^{\mathrm{D}^{\prime}}\right) \cdot\left(\boldsymbol{\sigma}^{\mathrm{N}}-\boldsymbol{\sigma}^{\mathrm{D}}\right) \cdot \hat{\mathbf{n}} \\
& +\int_{\Gamma_{\mathrm{s}}}\left(\mathbf{v}^{\mathrm{N}}-\mathbf{v}^{\mathrm{D}}\right) \cdot\left(\boldsymbol{\sigma}^{\mathrm{N}^{\prime}}-\boldsymbol{\sigma}^{\mathrm{D}^{\prime}}\right) \cdot \hat{\mathbf{n}} \\
= & \int_{\Gamma_{\mathrm{s}}} \mathbf{v}^{\mathrm{N}^{\prime}} \cdot\left(\boldsymbol{\sigma}^{\mathrm{N}}-\boldsymbol{\sigma}^{\mathrm{D}}\right) \cdot \hat{\mathbf{n}} \\
& -\int_{\Gamma_{\mathrm{s}}}\left(\mathbf{v}^{\mathrm{N}}-\mathbf{v}^{\mathrm{D}}\right) \cdot \boldsymbol{\sigma}^{\mathrm{D}^{\prime}} \cdot \hat{\mathbf{n}} .
\end{aligned}
$$

Minimizing / subject to the constraints $\mathcal{D P}$ and $\mathcal{N} \mathcal{P}$ requires $\mathrm{d} J=0$ for arbitrary $\mathbf{v}^{\mathrm{N}^{\prime}}$ and $\mathbf{v}^{\mathrm{D}^{\prime}}$ (and hence $\boldsymbol{\sigma}^{\mathrm{D}^{\prime}}$ ). This requires $\mathbf{v}^{\mathrm{N}}=\mathbf{v}^{\mathrm{D}}=\mathbf{f}$, and $\boldsymbol{\sigma}^{\mathrm{D}} \cdot \hat{\mathbf{n}}=\boldsymbol{\sigma}^{\mathrm{N}} \cdot \hat{\mathbf{n}}=\boldsymbol{\tau}$ on $\Gamma_{\mathrm{s}}$. In that case $J$ is zero from Equation (A3) while elsewhere it is positive, so the stationary point must be a minimum. The variational problem of minimizing $J$, subject to the constraints of $\mathcal{D P}$ and $\mathcal{N P}$, solves the inverse problem $\mathcal{I P}$.

To derive Equation (4), introduce the firstorder perturbations of the Neumann and Dirichlet problems by substituting perturbed quantities $\left(\mu+\mu^{\prime}, \beta+\beta^{\prime}, \mathbf{v}^{\mathrm{N}}+\mathbf{v}^{\mathrm{N}^{\prime}}\right.$, etc.) into $\mathcal{D P}$ and $\mathcal{N} \mathcal{P}$, subtracting the unperturbed equations, then neglecting terms that involve products of perturbations:

$\left(\mathcal{D} \mathcal{P}^{\prime}\right)\left\{\begin{aligned} \frac{1}{2}\left[\left(\nabla \mathbf{v}^{\prime}\right)+\left(\nabla \mathbf{v}^{\prime}\right)^{*}\right] & =\dot{\mathbf{e}}^{\prime} & & \text { in } \Omega, \\ \boldsymbol{\sigma}^{\prime}+p^{\prime} \mathbf{I} & =2 \mu \dot{\mathbf{e}}^{\prime}+2 \mu^{\prime} \mathbf{e}^{\mathrm{D}} & & \text { in } \Omega, \\ \operatorname{Trace}\left(\boldsymbol{\sigma}^{\prime}\right)+3 p^{\prime}=\nabla \cdot \mathbf{v}^{\prime} & =0 & & \text { in } \Omega, \\ \nabla \cdot \boldsymbol{\sigma}^{\prime} & =0 & & \text { in } \Omega, \\ \mathbf{v}^{\prime} & =0 & & \text { on } \Gamma_{\mathrm{s}}, \\ \boldsymbol{\sigma}^{\prime} \cdot \hat{\mathbf{n}}+\beta \mathbf{v}^{\prime}+\beta^{\prime} \mathbf{v}^{\mathrm{D}} & =0 & & \text { on } \Gamma_{\mathrm{b}} ;\end{aligned}\right.$

$\left(\mathcal{N} \mathcal{P}^{\prime}\right)\left\{\begin{aligned} \frac{1}{2}\left[\left(\nabla \mathbf{v}^{\prime}\right)+\left(\nabla \mathbf{v}^{\prime}\right)^{*}\right] & =\dot{\mathbf{e}}^{\prime} & & \text { in } \Omega, \\ \boldsymbol{\sigma}^{\prime}+p^{\prime} \mathbf{I} & =2 \mu \dot{\mathbf{e}}^{\prime}+2 \mu^{\prime} \dot{\mathbf{e}}^{\mathrm{N}} & & \text { in } \Omega, \\ \operatorname{Trace}\left(\boldsymbol{\sigma}^{\prime}\right)+3 p^{\prime}=\nabla \cdot \mathbf{v}^{\prime} & =0 & & \text { in } \Omega, \\ \nabla \cdot \boldsymbol{\sigma}^{\prime} & =0 & & \text { in } \Omega, \\ \boldsymbol{\sigma}^{\prime} \cdot \hat{\mathbf{n}} & =0 & & \text { on } \Gamma_{\mathrm{s}} \\ \boldsymbol{\sigma}^{\prime} \cdot \hat{\mathbf{n}}+\beta \mathbf{v}^{\prime}+\beta^{\prime} \mathbf{v}^{N} & =0 & & \text { on } \Gamma_{\mathrm{b}}\end{aligned}\right.$

with solutions $\left[\mathbf{v}^{\mathrm{D}^{\prime}}, \dot{\mathbf{e}}^{\mathrm{D}^{\prime}}, \boldsymbol{\sigma}^{\mathrm{D}^{\prime}}, p^{\mathrm{D}^{\prime}}\right]$ and $\left[\mathbf{v}^{\mathrm{N}^{\prime}}, \dot{\mathbf{e}}^{\mathrm{N}^{\prime}}, \boldsymbol{\sigma}^{\mathrm{N}^{\prime}}, p^{\mathrm{N}^{\prime}}\right]$, respectively.

The following useful result, with $(A, B) \in(D, N)$, uses Equations (A1) and (A2), and the relationship $\dot{\mathbf{e}}^{\mathrm{A}}: \boldsymbol{\sigma}^{\mathrm{B}^{\prime}}=$ $\boldsymbol{\sigma}^{\mathrm{A}}: \dot{\mathbf{e}}^{\mathrm{B}^{\prime}}+2 \mu^{\prime} \dot{\mathbf{e}}^{\mathrm{A}}: \dot{\mathbf{e}}^{\mathrm{B}}$, derived from $\mathcal{D P}, \mathcal{N} \mathcal{P}, \mathcal{D} \mathcal{P}^{\prime}$ and $\mathcal{N} \mathcal{P}^{\prime}$ :

$$
\begin{aligned}
\int_{\Gamma_{\mathrm{s}}} \mathbf{v}^{\mathrm{A}} \cdot \boldsymbol{\sigma}^{\mathrm{B}^{\prime}} \cdot \hat{\mathbf{n}} & =\int_{\Omega} \nabla \cdot \mathbf{v}^{\mathrm{A}} \cdot \boldsymbol{\sigma}^{\mathrm{B}^{\prime}}-\int_{\Gamma_{\mathrm{b}}} \mathbf{v}^{\mathrm{A}} \cdot \boldsymbol{\sigma}^{\mathrm{B}^{\prime}} \cdot \hat{\mathbf{n}} \\
& =\int_{\Omega} \mathbf{v}^{\mathrm{A}} \cdot \nabla \cdot \boldsymbol{\sigma}^{\mathrm{B}^{\prime}}+\int_{\Omega} \dot{\mathbf{e}}^{\mathrm{A}}: \boldsymbol{\sigma}^{\mathrm{B}^{\prime}} \\
& -\int_{\Gamma_{\mathrm{b}}} \mathbf{v}^{\mathrm{A}} \cdot \boldsymbol{\sigma}^{\mathrm{B}^{\prime}} \cdot \hat{\mathbf{n}} \\
& =\int_{\Omega} \mathbf{v}^{\mathrm{A}} \cdot \nabla \cdot \boldsymbol{\sigma}^{\mathrm{B}^{\prime}}+\int_{\Omega} \nabla \cdot \mathbf{v}^{\mathrm{B}^{\prime}} \cdot \boldsymbol{\sigma}^{\mathrm{A}} \\
& -\int_{\Omega} \mathbf{v}^{\mathrm{B}^{\prime}} \cdot \nabla \cdot \boldsymbol{\sigma}^{\mathrm{A}}+\int_{\Omega} 2 \mu^{\prime} \dot{\mathbf{e}}^{\mathrm{A}}: \dot{\mathbf{e}}^{\mathrm{B}} \\
& -\int_{\Gamma_{\mathrm{b}}} \mathbf{v}^{\mathrm{A}} \cdot \boldsymbol{\sigma}^{\mathrm{B}^{\prime}} \cdot \hat{\mathbf{n}} \\
& =\int_{\Omega} \mathbf{v}^{\mathrm{A}} \cdot \nabla \cdot \boldsymbol{\sigma}^{\mathrm{B}^{\prime}}-\int_{\Omega} \mathbf{v}^{\mathrm{B}^{\prime}} \cdot \nabla \cdot \boldsymbol{\sigma}^{\mathrm{A}} \\
& -\int_{\Gamma_{\mathrm{b}}} \mathbf{v}^{\mathrm{A}} \cdot \boldsymbol{\sigma}^{\mathrm{B}^{\prime}} \cdot \hat{\mathbf{n}}+\int_{\Gamma_{\mathrm{b}}} \mathbf{v}^{\mathrm{B}^{\prime}} \cdot \boldsymbol{\sigma}^{\mathrm{A}} \cdot \hat{\mathbf{n}} \\
& +\int_{\Gamma_{\mathrm{s}}} \mathbf{v}^{\mathrm{B}^{\prime}} \cdot \boldsymbol{\sigma}^{\mathrm{A}} \cdot \hat{\mathbf{n}}+\int_{\Omega} 2 \mu^{\prime} \dot{\mathbf{e}}^{\mathrm{A}}: \dot{\mathbf{e}}^{\mathrm{B}} .
\end{aligned}
$$

Applying Equation (A7) to each term in Equation (A4), and making substitutions from $\mathcal{N} \mathcal{P}, \mathcal{D P}, \mathcal{D P} \mathcal{P}^{\prime}$ and $\mathcal{N} \mathcal{P}^{\prime}$ (i.e. Equations (1), (2), (A5) and (A6)) provides Equation (4), after some algebra and cancellation of terms. 